\title{
EXISTENCE OF SOLUTION TO A CRITICAL EQUATION WITH VARIABLE EXPONENT
}

\author{
Julián Fernández Bonder, Nicolas Saintier and Analía Silva \\ IMAS - CONICET and Universidad de Buenos Aires, Departamento de Matemática, FCEyN \\ Ciudad Universitaria, Pabellón I (1428) Buenos Aires, Argentina; jfbonder@dm.uba.ar \\ Universidad Nacional de General Sarmiento, Instituto de Ciencias \\ Juan María Gutierrez 1150 Los Polvorines, Pcia de Bs. As., Argentina; nsaintie@ungs.edu.ar \\ and Universidad de Buenos Aires, Departamento de Matemática, FCEyN \\ Ciudad Universitaria, Pabellón I (1428) Buenos Aires, Argentina; nsaintie@dm.uba.ar \\ IMAS - CONICET and Universidad de Buenos Aires, Departamento de Matemática, FCEyN \\ Ciudad Universitaria, Pabellón I (1428) Buenos Aires, Argentina; asilva@dm.uba.ar
}

\begin{abstract}
In this paper we study the existence problem for the $p(x)$-Laplacian operator with a nonlinear critical source. We find a local condition on the exponents ensuring the existence of a nontrivial solution that shows that the Pohozaev obstruction does not holds in general in the variable exponent setting. The proof relies on the Concentration-Compactness Principle for variable exponents and the Mountain Pass Theorem.
\end{abstract}

\section{Introduction}

In this paper we address the existence problem for the $p(x)$-Laplace operator with a source that has critical growth in the sense of the Sobolev embeddings. To be precise, we consider the equation

$$
\begin{cases}-\Delta_{p(\cdot)} u+h|u|^{p(\cdot)-2} u=|u|^{q(\cdot)-2} u & \text { in } U \\ u=0 & \text { on } \partial U\end{cases}
$$

where $U \subset \mathbf{R}^{n}$ is a smooth bounded domain, $p, q: U \rightarrow[1, \infty)$ are log-Hölder continuous functions such that $1<\inf _{U} p \leq \sup _{U} p<n$ and $1 \leq q(x) \leq p^{*}(x):=$ $n p(x) /(n-p(x)), x \in U$.

The $p(x)$-Laplacian operator $\Delta_{p(\cdot)}$ is defined, as usual, as

$$
\Delta_{p(\cdot)} u:=\operatorname{div}\left(|\nabla u|^{p(\cdot)-2} \nabla u\right) .
$$

This operator appears in the study of the so-called electrorheological fluids. We refer to the monograph by Rǔžička, [25], and its references, for a detailed account. In particular, after some simplifications, the modelling of these fluids lead to solve

$$
\begin{cases}-\Delta_{p(\cdot)} u=f(\cdot, u, \nabla u) & \text { in } U \\ u=0 & \text { on } \partial U\end{cases}
$$

doi:10.5186/aasfm.2012.3743

2010 Mathematics Subject Classification: Primary 35J92, 35B33.

Key words: Sobolev embedding, variable exponents, critical exponents, concentration compactness. 
for some nonlinear source $f$. In most cases, the source term is taken to be only dependent on $u$ and so, in order for the usual variational techniques to work, one needs a control on the growth of $f$ given by the Sobolev embedding.

When the growth of $f$ is subcritical in the sense of the Sobolev embedding the existence of solution follows easily by applying standard procedures of the calculus of variations (see e.g. [6, 10, 20, 21, 22] and many others). On the other hand, when the source term has critical growth, there are only a few results on the existence of solutions for (1.2) that we will review thoroughly later. Let us just notice for the moment that these results only provide global existence conditions. This strongly contrasts with the constant exponent case that has been widely studied since Aubin and Brezis-Nirenberg's seminal works $[2,5]$, and for which it is generally possible to provide existence conditions that are local in the sense that they involve the behaviour of the coefficients of the equations (and possibly some relevant geometric quantities) only in a neighborhood of a point. Our main purpose in this paper is to provide local existence conditions for the equation (1.1).

In order to study (1.1) by means of variational methods, we introduce the functional $J: W_{0}^{1, p(\cdot)}(U) \rightarrow \mathbf{R}$ defined by

$$
J(u):=\int_{U} \frac{1}{p(x)}\left(|\nabla u|^{p(x)}+h(x)|u|^{p(x)}\right) d x-\int_{U} \frac{1}{q(x)}|u|^{q(x)} d x .
$$

This functional is naturally associated to (1.1) in the sense that a weak solution of (1.1) is a critical point of $J$. We refer to Section 2 for the definition and some elementary properties of variable exponent spaces.

We need to assume that the smooth function $h$ is such that the functional

$$
I(u):=\int_{U} \frac{1}{p(x)}\left(|\nabla u|^{p(x)}+h(x)|u|^{p(x)}\right) d x
$$

is coercive in the sense that the norm

$$
\|u\|:=\inf \left\{\lambda>0: \int_{U} \frac{|\nabla u|^{p(x)}+h(x)|u(x)|^{p(x)}}{\lambda^{p(x)}} d x \leq 1\right\}
$$

is equivalent to the usual norm of $W_{0}^{1, p(\cdot)}(U)$. Notice that a sufficient condition is

$$
\inf _{U} h>-\lambda_{1} \quad \text { where } \lambda_{1}=\inf _{u \in W_{0}^{1, p(.)}(U)} \frac{\int_{U}|\nabla u|^{p(x)} d x}{\int_{U}|u|^{p(x)} d x}>0 .
$$

In particular $h$ can be negative.

When $\inf _{U} q>\sup _{U} p$, it is easy to show that $J$ satisfies the geometric assumptions of the Mountain-Pass Theorem (cf. Section 4). Hence if we assume moreover that the exponent $q$ is subcritical in the sense that

$$
\inf _{U}\left(p^{*}-q\right)>0
$$

which implies that the immersion $W_{0}^{1, p(\cdot)}(U) \hookrightarrow L^{q(\cdot)}(U)$ is compact, then $J$ satisfies the Palais-Smale condition, and the existence of a nontrivial solution to (1.1) follows easily.

When (1.5) is violated, the immersion $W_{0}^{1, p(\cdot)}(U) \hookrightarrow L^{q(\cdot)}(U)$ does not need to be compact and so the Palais-Smale condition may fail. The existence of a non-trivial solution to (1.1) is then a non-trivial problem. 
We denote by

$$
\mathcal{A}:=\left\{x \in U: q(x)=p^{*}(x)\right\}
$$

the critical set. We will assume in this work that this critical set is nonempty.

In [23] the authors prove that if $\mathcal{A}$ is small and there exists a control on the rate of how $q$ reaches the critical value $p^{*}$, then the immersion $W_{0}^{1, p(\cdot)}(U) \hookrightarrow L^{q(\cdot)}(U)$ remains compact, and so the usual techniques can be applied. When the immersion fails to be compact they prove that if the subcriticality set $U \backslash \mathcal{A}$ contains a sufficiently large ball, then (1.1) with $h=0$ has a nonnegative solution.

In [14], problem (1.1) is studied with $h=0$ and with a subcritical perturbation. In this work the authors generalize the Concentration-Compactness Principle (CCP) of Lions to the variable exponent case and prove that if the subcritical perturbation is large enough on the critical set, the Palais-Smale condition is verified and so the existence of a nontrivial solution follows. See also [15] where similar results were obtained independently.

In [27], using the CCP of $[14,15]$, a multiplicity problem for (1.1) with $h=0$ and a nonsymmetric subcritical perturbation is analyzed.

More recently, the authors in [12] studied the best Sobolev constant $S(p(\cdot), q(\cdot), U)$ corresponding to the embedding $W_{0}^{1, p(\cdot)}(U) \hookrightarrow L^{q(\cdot)}(U)$, namely

$$
S(p(\cdot), q(\cdot), U)=\inf _{u \in W_{0}^{1, p(\cdot)}(U)} \frac{\|\nabla u\|_{L^{p(\cdot)}(U)}}{\|u\|_{L^{q(\cdot)}(U)}} .
$$

Using a refinement of the CCP proved in [14], they gave sufficient conditions for the existence of an extremal for $S(p(\cdot), q(\cdot), U)$, and so the existence of a solution to (1.1) with $h=0$ follows.

We remark that in [12] a bit more than log-Hölder regularity on the exponents $p$ and $q$ is required, i.e. it is needed that $p(\cdot), q(\cdot): \Omega \rightarrow[1,+\infty)$ are continuous functions with modulus of continuity $\rho(t)$ such that

$$
\rho(t) \log (1 / t) \rightarrow 0 \quad \text { as } t \rightarrow 0+.
$$

The study of (1.1) posed in the whole $\mathbf{R}^{n}$ is analyzed in $[1,16]$. In those works the authors studied the problem in the case where $p, q$ and $h$ are radial functions and give somewhat restrictive conditions to ensure the existence of a nontrivial radial solution.

From now on we will assume that

$$
\sup _{U} p<\inf _{U} q .
$$

Our first result provides a condition for the functional $J$ defined by (1.3) to satisfy the Palais-Smale condition.

Theorem 1.1. Assume that the exponents $p$ and $q$ verify (1.8) and, moreover, that the critical set $\mathcal{A}$ given in (1.6) is nonempty. Then, the functional $J$ satisfies the Palais-Smale condition at level $c \in\left(0, \frac{1}{n} S^{n}\right)$ where

$$
S:=\inf _{x \in \mathcal{A}} \lim _{\epsilon \rightarrow 0} S\left(p(\cdot), q(\cdot), B_{\epsilon}(x)\right),
$$

and $S\left(p(\cdot), q(\cdot), B_{\epsilon}(x)\right)$ stands for the best Sobolev constant for the domain $B_{\epsilon}(x)$ defined in a similar way as in (1.7). 
The proof of Theorem 1.1 relies on a precise computation of the constants in the CCP recently proved in [12].

As a corollary, we can apply the Mountain-Pass Theorem to obtain the following necessary existence condition:

Theorem 1.2. Under the same assumptions of Theorem 1.1, if there exists $v \in$ $W_{0}^{1, p(\cdot)}(U)$ such that

$$
\sup _{t>0} J(t v)<\frac{1}{n} S^{n}
$$

then (1.1) has a non-trivial nonnegative solution.

Eventually the following result provide a sufficient local condition for (1.11) to hold:

Theorem 1.3. Assume that the infimum in the definition (1.10) of $S$ is attained at a point $x_{0} \in \mathcal{A}$ such that $x_{0}$ is a local minimum of $p$ and a local maximum of $q$. In particular,

$$
-\Delta p\left(x_{0}\right) \leq 0 \leq-\Delta q\left(x_{0}\right) .
$$

Assume, moreover, that $p, q$ are $C^{2}$ in a neighborhood of $x_{0}$, and that $h\left(x_{0}\right)<0$ if $1<p\left(x_{0}\right)<2(n \geq 4)$, or if $2 \leq p\left(x_{0}\right)<\sqrt{n}(n \geq 5)$, that at least one of the two inequalities in (1.12) is strict, but $h\left(x_{0}\right)$ is arbitrary. Under these assumptions (1.11) holds. In particular, (1.1) has a non-trivial nonnegative solution.

In the constant exponent case, the well known Pohozaev obstruction [24] affirms that if $h \geq 0$ and $U$ is starshaped then there are no (positive) solutions to (1.1). Our result shows that for variable $p$ and $q$ and $p(x) \geq 2$ this does not need to be the case, showing a stricking difference between the constant exponent case and the variable exponent one.

\section{Preliminaries on variable exponent spaces}

In this section we review some preliminary results regarding Lebesgue and Sobolev spaces with variable exponent. All of these results and a comprehensive study of these spaces can be found in [8].

Consider a function $p: U \rightarrow[1,+\infty] \log$-Hölder continuous in the sense that

$$
|p(x)-p(y)| \leq \frac{C}{|\log | x-y||}, \quad \text { for } x, y \in U, x \neq y
$$

for some constant $C>0$. This regularity assumptions is not needed to define the Lebesgue and Sobolev spaces with variable $p$ but turns out to be very useful for these Sobolev spaces to enjoy all the usual properties like Sobolev embeddings, Poincaré inequality and so on. We will therefore assume it from now for simplicity.

The variable exponent Lebesgue space $L^{p(\cdot)}(U)$ is defined by

$$
L^{p(\cdot)}(U)=\left\{u \in L_{\mathrm{loc}}^{1}(U): \int_{U}|u(x)|^{p(x)} d x<\infty\right\} .
$$

This space is endowed with the norm

$$
\|u\|_{L^{p(\cdot)}(U)}=\inf \left\{\lambda>0: \int_{U}\left|\frac{u(x)}{\lambda}\right|^{p(x)} d x \leq 1\right\} .
$$


The variable exponent Sobolev space $W^{1, p(\cdot)}(U)$ is defined by

$$
W^{1, p(\cdot)}(U)=\left\{u \in W_{\mathrm{loc}}^{1,1}(U): u \in L^{p(\cdot)}(U) \text { and }|\nabla u| \in L^{p(\cdot)}(U)\right\} .
$$

The corresponding norm for this space is

$$
\|u\|_{W^{1, p(\cdot)}(U)}=\|u\|_{L^{p(\cdot)}(U)}+\|\nabla u\|_{L^{p(\cdot)}(U)} .
$$

Define $W_{0}^{1, p(\cdot)}(U)$ as the closure of $C_{c}^{\infty}(U)$ with respect to the $W^{1, p(\cdot)}(U)$ norm. The spaces $L^{p(\cdot)}(U), W^{1, p(\cdot)}(U)$ and $W_{0}^{1, p(\cdot)}(U)$ are separable and reflexive Banach spaces

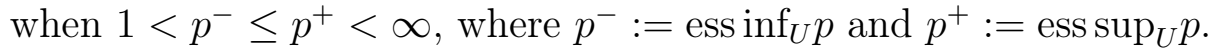

As usual, we denote the conjugate exponent of $p(x)$ by $p^{\prime}(x)=p(x) /(p(x)-1)$ and the Sobolev exponent by

$$
p^{*}(x)= \begin{cases}\frac{N p(x)}{N-p(x)} & \text { if } p(x)<N \\ \infty & \text { if } p(x) \geq N\end{cases}
$$

The following result is proved in [18] (see also [11] and [8], pp. 79, Lemma 3.2.20 $(3.2 .23))$.

Proposition 2.1. (Hölder-type inequality) Let $f \in L^{p(\cdot)}(U)$ and $g \in L^{q(\cdot)}(U)$. Then the following inequality holds

$$
\|f g\|_{L^{s(\cdot)}(U)} \leq\left(\left(\frac{s}{p}\right)^{+}+\left(\frac{s}{q}\right)^{+}\right)\|f\|_{L^{p(\cdot)}(U)}\|g\|_{L^{q(\cdot)}(U)},
$$

where

$$
\frac{1}{s(x)}=\frac{1}{p(x)}+\frac{1}{q(x)} .
$$

The Sobolev embedding Theorem is also proved in [11], Theorem 2.3.

Proposition 2.2. (Sobolev embedding) Let $q: U \in[1,+\infty)$ be a measurable function such that $1 \leq q(x) \leq p^{*}(x)<\infty$ for all $x \in \bar{U}$. Then there is a continuous embedding

$$
W^{1, p(\cdot)}(U) \hookrightarrow L^{q(\cdot)}(U) .
$$

Moreover, if $\inf _{U}\left(p^{*}-q\right)>0$ then, the embedding is compact.

As in the constant exponent spaces, Poincaré inequality holds true (see [8], pp. 249, Theorem 8.2.4).

Proposition 2.3. (Poincaré inequality) There is a constant $C>0, C=C(U)$, such that

$$
\|u\|_{L^{p(\cdot)}(U)} \leq C\|\nabla u\|_{L^{p(\cdot)}(U)},
$$

for all $u \in W_{0}^{1, p(\cdot)}(U)$.

It follows from the Poincaré inequality that $\|\nabla u\|_{L^{p(\cdot)}(U)}$ and $\|u\|_{W^{1, p(\cdot)}(U)}$ are equivalent norms on $W_{0}^{1, p(\cdot)}(U)$, in particular.

Throughout this paper the following notation will be used: Given $q: U \rightarrow \mathbf{R}$ bounded, we denote

$$
q^{+}:=\sup _{U} q(x), \quad q^{-}:=\inf _{U} q(x) .
$$

The following proposition is also proved in [11] and it will be most usefull (see also [8], Chapter 2, Section 1). 
Proposition 2.4. Set $\rho(u):=\int_{U}|u(x)|^{p(x)} d x$. For $u \in L^{p(\cdot)}(U)$ and $\left\{u_{k}\right\}_{k \in \mathbf{N}} \subset$ $L^{p(\cdot)}(U)$, we have

$$
\begin{aligned}
& u \neq 0 \Rightarrow\left(\|u\|_{L^{p(\cdot)}(U)}=\lambda \Leftrightarrow \rho\left(\frac{u}{\lambda}\right)=1\right), \\
& \|u\|_{L^{p(\cdot)}(U)}<1(=1 ;>1) \Leftrightarrow \rho(u)<1(=1 ;>1), \\
& \|u\|_{L^{p(\cdot)}(U)}>1 \Rightarrow\|u\|_{L^{p(\cdot)}(U)}^{p^{-}} \leq \rho(u) \leq\|u\|_{L^{p(\cdot)}(U)}^{p^{+}}, \\
& \|u\|_{L^{p(\cdot)}(U)}<1 \Rightarrow\|u\|_{L^{p(\cdot)}(U)}^{p^{+}} \leq \rho(u) \leq\|u\|_{L^{p(\cdot)}(U)}^{p^{-}}, \\
& \lim _{k \rightarrow \infty}\left\|u_{k}\right\|_{L^{p(\cdot)}(U)}=0 \Leftrightarrow \lim _{k \rightarrow \infty} \rho\left(u_{k}\right)=0, \\
& \lim _{k \rightarrow \infty}\left\|u_{k}\right\|_{L^{p(\cdot)}(U)}=\infty \Leftrightarrow \lim _{k \rightarrow \infty} \rho\left(u_{k}\right)=\infty .
\end{aligned}
$$

The following Lemma is the extension to variable exponents of the well-known Brezis-Lieb Lemma (see [4]). The proof is analogous to that of [4]. See Lemma 3.4 in [14].

Lemma 2.5. Let $f_{n} \rightarrow f$ a.e. and $f_{n} \rightarrow f$ in $L^{p(\cdot)}(U)$ then

$$
\lim _{n \rightarrow \infty}\left(\int_{U}\left|f_{n}\right|^{p(x)} d x-\int_{U}\left|f-f_{n}\right|^{p(x)} d x\right)=\int_{U}|f|^{p(x)} d x
$$

For much more on these spaces, we refer to [8].

\section{Proof of Theorem 1.1}

In this section we verify that the functional $J$ defined by (1.3) satisfies the PalaisSmale condition (PS for short) for energy levels below the critical one $\frac{1}{n} S^{n}$.

Recall that $J$ is said to satisfy the PS condition if any PS sequence for $J$ has a strongly converging subsequence, where a sequence $\left\{u_{k}\right\}_{k \in \mathbf{N}} \subset W_{0}^{1, p(\cdot)}(U)$ is called a PS-sequence for $J$ if

i. The sequence $\left\{J\left(u_{k}\right)\right\}_{k \in \mathbf{N}}$ is bounded, and

ii. $D J\left(u_{k}\right) \rightarrow 0$ strongly in the dual space $\left(W_{0}^{1, p(\cdot)}(U)\right)^{\prime}$.

The scheme of the proof is classical (see e.g. [26]) but relies on a version of Lions' concentration-compactness principle adapted to the variable exponent setting in [14] and then refined in [12].

Let $\left\{u_{k}\right\}_{k \in \mathbf{N}} \subset W_{0}^{1, p(\cdot)}(U)$ be a PS-sequence for $J$. Recalling that the functional $I$ defined by (1.4) is assumed to be coercive, it then follows that $\left\{u_{k}\right\}_{k \in \mathbf{N}}$ is bounded in $W_{0}^{1, p(\cdot)}(U)$. In fact, for $k$ large, we have that

$$
\begin{aligned}
c+1 & \geq J\left(u_{k}\right)-\frac{1}{q^{-}}\left\langle D J\left(u_{k}\right), u_{k}\right\rangle \\
& \geq\left(\frac{1}{p^{+}}-\frac{1}{q^{-}}\right) \int_{U}\left|\nabla u_{k}\right|^{p(x)}+h(x)\left|u_{k}\right|^{p(x)} d x-\int_{U}\left(\frac{1}{q(x)}-\frac{1}{q^{-}}\right)\left|u_{k}\right|^{q(x)} d x \\
& \geq\left(\frac{1}{p^{+}}-\frac{1}{q^{-}}\right) \int_{U}\left|\nabla u_{k}\right|^{p(x)}+h(x)\left|u_{k}\right|^{p(x)} d x .
\end{aligned}
$$

from where the claim follows recalling assumption (1.9).

We may thus assume that $u_{k} \rightarrow u$ weakly in $W_{0}^{1, p(\cdot)}(U)$. We claim that $u$ turns out to be a weak solution to (1.1). In fact, since $u_{k} \rightarrow u$ weakly in $W_{0}^{1, p(\cdot)}(U)$ it 
follows that

$$
\begin{aligned}
& \left|\nabla u_{k}\right|^{p(\cdot)-2} \nabla u_{k} \rightarrow|\nabla u|^{p(\cdot)-2} \nabla u \quad \text { weakly in } L^{p^{\prime}(\cdot)}(U), \\
& \left|u_{k}\right|^{p(\cdot)-2} u_{k} \rightarrow|u|^{p(\cdot)-2} u \quad \text { weakly in } L^{p^{\prime}(\cdot)}(U), \\
& \left|u_{k}\right|^{q(\cdot)-2} u_{k} \rightarrow|u|^{q(\cdot)-2} u \quad \text { weakly in } L^{q^{\prime}(\cdot)}(U) .
\end{aligned}
$$

So

$$
\begin{aligned}
0 & =\lim _{k \rightarrow \infty}\left\langle D J\left(u_{k}\right), \phi\right\rangle \\
& =\lim _{k \rightarrow \infty} \int_{U}\left|\nabla u_{k}\right|^{p(x)-2} \nabla u_{k} \nabla \phi+h\left|u_{k}\right|^{p(x)-2} u_{k} \phi d x-\int_{U}\left|u_{k}\right|^{q(x)-2} u_{k} \phi \\
& =\int_{U}|\nabla u|^{p(x)-2} \nabla u \nabla \phi+h|u|^{p(x)-2} u \phi d x-\int_{U}|u|^{q(x)-2} u \phi,
\end{aligned}
$$

for every $\phi \in C_{0}^{\infty}(U)$. This proves that $u$ is a weak solution of (1.1).

By the CCP for variable exponents (see [14] and the refinement proved in [12]) it holds that

$$
\begin{aligned}
& \left|u_{k}\right|^{q(\cdot)} \rightarrow \nu=|u|^{q(\cdot)}+\sum_{i \in I} \nu_{i} \delta_{x_{i}} \quad \text { weakly in the sense of measures, } \\
& \left|\nabla u_{k}\right|^{p(\cdot)} \rightarrow \mu \geq|\nabla u|^{p(\cdot)}+\sum_{i \in I} \mu_{i} \delta_{x_{i}} \quad \text { weakly in the sense of measures, } \\
& S \nu_{i}^{1 / p^{*}\left(x_{i}\right)} \leq \mu_{i}^{1 / p\left(x_{i}\right)}
\end{aligned}
$$

where $I$ is a finite set, $\left\{\nu_{i}\right\}_{i \in I}$ and $\left\{\mu_{i}\right\}_{i \in I}$ are positive numbers and the points $\left\{x_{i}\right\}_{i \in I}$ belong to the critical set $\mathcal{A}$ defined in (1.6).

It is not difficult to check (arguing as in (3.1)) that $v_{k}:=u_{k}-u$ is PS-sequence for $\tilde{J}(v):=J(v)-\int_{U} \frac{1}{p(x)} h|v|^{p(x)}$. Now, by Lemma 2.5 we get

$$
\begin{aligned}
J\left(u_{k}\right)-J(u) & =\int_{U} \frac{1}{p(x)}\left[\left|\nabla v_{k}\right|^{p(x)}+h\left|v_{k}\right|^{p(x)}\right] d x-\int_{U} \frac{1}{q(x)}\left|v_{k}\right|^{q(x)} d x+o(1) \\
& =\tilde{J}\left(v_{k}\right)+\int_{U} \frac{1}{p(x)} h\left|v_{k}\right|^{p(x)} d x+o(1)=\tilde{J}\left(v_{k}\right)+o(1) .
\end{aligned}
$$

Since $u$ is a weak solution of (1.1), and since $p^{+}<q^{-}$,

$$
\begin{aligned}
J(u) & \geq \frac{1}{p^{+}} \int_{U}\left(|\nabla u|^{p(x)}+h(x)|u|^{p(x)}\right) d x-\frac{1}{q^{-}} \int_{U}|u|^{q(x)} d x \\
& =\left(\frac{1}{p^{+}}-\frac{1}{q^{-}}\right) \int_{U}|u|^{q(x)} d x \geq 0 .
\end{aligned}
$$

Therefore,

$$
J\left(u_{k}\right) \geq \tilde{J}\left(v_{k}\right)+o(1) .
$$

Let $\phi \in C_{c}^{\infty}(U)$. As $D \tilde{J}\left(v_{k}\right) \rightarrow 0$, we have

$$
\begin{aligned}
o(1) & =\left\langle D \tilde{J}\left(v_{k}\right), v_{k} \phi\right\rangle \\
& =\int_{U}\left|\nabla v_{k}\right|^{p(x)} \phi d x-\int_{U}\left|v_{k}\right|^{q(x)} \phi d x+\int_{U}\left|\nabla v_{k}\right|^{p(x)-2} \nabla v_{k} \nabla \phi v_{k} d x \\
& =A-B+C .
\end{aligned}
$$


Since $v_{k} \rightarrow 0$ weakly in $W_{0}^{1, p(\cdot)}(U)$ it is easy to see that $C \rightarrow 0$ as $k \rightarrow \infty$. By means of Lemma 2.5 it follows that

$$
A \rightarrow \int_{U} \phi d \tilde{\mu} \quad \text { and } \quad B \rightarrow \int_{U} \phi d \tilde{\nu}
$$

where $\tilde{\mu}=\mu-|\nabla u|^{p(x)}$ and $\tilde{\nu}=\nu-|u|^{q(x)}$. So we conclude that $\tilde{\mu}=\tilde{\nu}$. In particular, $\nu_{i} \geq \mu_{i}(i \in I)$ from where we obtain that $\nu_{i} \geq S^{n}$. Hence

$$
\begin{aligned}
c & =\lim _{k \rightarrow \infty} J\left(u_{k}\right) \geq \lim _{k \rightarrow \infty} \tilde{J}\left(v_{k}\right)=\int \frac{1}{p(x)} d \tilde{\mu}-\int \frac{1}{q(x)} d \tilde{\nu} \\
& =\int\left(\frac{1}{p(x)}-\frac{1}{q(x)}\right) d \tilde{\nu}=\sum_{i \in I}\left(\frac{1}{p\left(x_{i}\right)}-\frac{1}{p^{*}\left(x_{i}\right)}\right) \nu_{i} \geq \#(I) \frac{1}{n} S^{n} .
\end{aligned}
$$

We deduce that if $c<\frac{1}{n} S^{n}$ then $I$ must be empty implying that $u_{k} \rightarrow u$ strongly in $W_{0}^{1, p(\cdot)}(U)$.

\section{Proof of Theorem 1.2}

The proof of Theorem 1.2 is an immediate consequence of the Mountain Pass Theorem, Theorem 1.1 and assumption (1.11).

In fact, it suffices to verify that $J$ has the Mountain Pass geometry in the sense that

(1) $J(0)=0$ (this is obvious),

(2) there exist $r, \rho>0$ such that $J(v) \geq \rho$ if $\|v\|_{W_{0}^{1, p(\cdot)}(U)}=r$, and

(3) there exist some $v,\|v\|_{W_{0}^{1, p(\cdot)}(U)}>r$, such that $J(v) \leq 0$.

Concerning the latter condition notice that, given some $u \not \equiv 0$, we have that $J(t u)<0$ for $t>0$ big enough. Indeed, for $t>1$,

$$
\begin{aligned}
J(t u) & =\int_{U} \frac{t^{p(x)}}{p(x)}\left(|\nabla u|^{p(x)}+h(x)|u|^{p(x)}\right) d x-\int_{U} \frac{t^{q(x)}}{q(x)}|u|^{q(x)} d x \\
& \leq t^{p^{+}} I(u)-t^{q^{-}} \int_{U} \frac{1}{q(x)}|u|^{q(x)} d x,
\end{aligned}
$$

which tends to $-\infty$ as $t \rightarrow+\infty$ since $q^{-}>p^{+}$.

It remains to verify condition (2). If $\|v\|_{W_{0}^{1, p(\cdot)}(U)}=r$ small enough, then

$$
\int_{U}|\nabla v|^{p(x)}+h|v|^{p(x)} d x \geq c\|v\|_{W_{0}^{1, p(\cdot)}(U)}^{p^{+}}
$$

and

so

$$
\|v\|_{L^{q(\cdot)}(U)} \leq C\|v\|_{W_{0}^{1, p(\cdot)}(U)}=C r<1
$$

$$
\int_{U}|v|^{q(x)} d x \leq C\|v\|_{W_{0}^{1, p(\cdot)}(U)}^{q^{-}} .
$$

Therefore

$$
J(v) \geq \frac{c}{p^{+}} r^{p^{+}}-\frac{C}{q^{-}} r^{q^{-}}>0,
$$

since $p^{+}<q^{-}$. This completes the proof. 


\section{Proof of Theorem 1.3}

Let $x_{0} \in \mathcal{A}$ be such that

$$
S:=\inf _{x \in \mathcal{A}} \lim _{\epsilon \rightarrow 0} S\left(p(\cdot), q(\cdot), B_{\epsilon}(x)\right)=\lim _{\epsilon \rightarrow 0} S\left(p(\cdot), q(\cdot), B_{\epsilon}\left(x_{0}\right)\right) .
$$

For ease of notation we assume that $x_{0}=0$, write $p=p(0)$ and observe that $q=q(0)=p^{*}$. From Theorem 6.1 in [12], we have that if 0 is a local maximum of $q$ and a local minimum of $p$, then

$$
S=\lim _{\epsilon \rightarrow 0} S\left(p(\cdot), q(\cdot), B_{\epsilon}(0)\right)=K(n, p)^{-1},
$$

where $K(n, p)$ is the best constant in the Sobolev inequality in $\mathbf{R}^{n}$, i.e.,

$$
K(n, p)^{-1}=\inf _{v \in W^{1, p}\left(\mathbf{R}^{n}\right)} \frac{\|\nabla v\|_{L^{p}\left(\mathbf{R}^{n}\right)}}{\|v\|_{L^{p^{*}}\left(\mathbf{R}^{n}\right)}}
$$

Let $U$ be an extremal for the constant $K(n, p)$. That is, $U$ verifies

$$
K(n, p)^{-1}=\frac{\|\nabla U\|_{L^{p}\left(\mathbf{R}^{n}\right)}}{\|U\|_{L^{p^{*}}\left(\mathbf{R}^{n}\right)}} .
$$

It is well known, see $[2,28]$, that $U$ can be given by the formula

$$
U(x)=\left(1+|x|^{\frac{p}{p-1}}\right)^{-\frac{n-p}{p}} .
$$

Moreover, any extremal for $K(n, p)$ is obtained by a translation and a dilation of $U$ in the form

$$
U_{\epsilon, x_{0}}(x)=\epsilon^{-\frac{n-p}{p}} U\left(\left(x-x_{0}\right) / \epsilon\right) .
$$

Given $\delta>0$ small we take a cut-off function $\eta \in C_{c}^{\infty}\left(B_{2 \delta},[0,1]\right)$ such that $\eta \equiv 1$ in $B_{\delta}$. We then consider the test-function

$$
u_{\epsilon}(x)=U_{\epsilon, 0}(x) \eta(x) .
$$

For this test function we have:

Proposition 5.1. Assume that 0 is a critical point of $p$ and $q$. We have

- If $p \leq \frac{n}{2}$,

$$
\int_{\mathbf{R}^{n}} f(x) u_{\epsilon}^{q(x)} d x=A_{0}+A_{1} \epsilon^{2} \ln \epsilon+o\left(\epsilon^{2} \ln \epsilon\right)
$$

with

$$
A_{0}=f(0) \int_{\mathbf{R}^{n}} U^{p^{*}} d x, \quad A_{1}=-\frac{n-p}{p} \frac{f(0)}{2} \int_{\mathbf{R}^{n}} U^{p^{*}}\left(D^{2} q(0) x, x\right) d x .
$$

- If $p<\min \left\{\sqrt{n}, \frac{n+2}{3}\right\}$,

$$
\int_{\mathbf{R}^{n}} f(x)\left|\nabla u_{\epsilon}\right|^{p(x)} d x=B_{0}+B_{1} \epsilon^{2} \ln \epsilon+o\left(\epsilon^{2} \ln \epsilon\right)
$$

with

$$
B_{0}=f(0) \int_{\mathbf{R}^{n}}|\nabla U|^{p} d x, \quad B_{1}=-\frac{n}{p} \frac{f(0)}{2} \int_{\mathbf{R}^{n}}|\nabla U|^{p}\left(D^{2} p(0) x, x\right) d x .
$$


- If $p<\sqrt{n}$,

$$
\int_{\mathbf{R}^{n}} f(x)\left|u_{\epsilon}\right|^{p(x)} d x=C_{0} \epsilon^{p}+o\left(\epsilon^{p}\right) \quad \text { with } \quad C_{0}=f(0) \int_{\mathbf{R}^{n}} U^{p} d x .
$$

Remark 5.2. Observe that if $g(x)$ is a radial function, then

$$
\int_{\mathbf{R}^{n}} g(x)(A x, x) d x=\operatorname{tr}(A) \int_{\mathbf{R}^{n}} g(x) x_{1}^{2} d x=\frac{\operatorname{tr}(A)}{n} \int_{\mathbf{R}^{n}} g(x)|x|^{2} d x,
$$

for any $A \in \mathbf{R}^{n \times n}$ (with adequate decaying assumptions at infinity on $g$ ). In fact, this is a consequence of the fact that, for $i \neq j$,

$$
\int_{\mathbf{R}^{n}} g(x) x_{i} x_{j} d x=0 .
$$

With this observation, we easily conclude that

$$
A_{1}=-\frac{f(0)}{p^{*}} \Delta q(0) \int_{\mathbf{R}^{n}} U^{p^{*}}|x|^{2} d x
$$

and

$$
B_{1}=-\frac{f(0)}{2 p} \Delta p(0) \int_{\mathbf{R}^{n}}|\nabla U|^{p}|x|^{2} d x .
$$

We postpone the proof of this proposition to Section 6 .

As $U$ is an extremal for $K(n, p)$ it follows that $U$ verifies

$$
-\Delta_{p} U=\frac{K(n, p)^{-p}}{\|U\|_{L^{p^{*}}\left(\mathbf{R}^{n}\right)}^{p^{*}}} U^{p^{*}-1}=C U^{p^{*}-1} .
$$

Then $V=C^{\frac{1}{p^{*}-p}} U=\frac{K(n, p)^{-\frac{n-p}{p}}}{\|U\|_{p^{*}}} U$ solves $-\Delta_{p} V=V^{p^{*}-1}$ and satisfy

$$
\|\nabla V\|_{L^{p}\left(\mathbf{R}^{n}\right)}=K(n, p)^{-n / p} .
$$

Consider the test function

$$
v_{\epsilon}(x)=\epsilon^{-\frac{n-p}{p}} V(x / \epsilon) \eta(x)=C^{\frac{1}{p^{*}-p}} u_{\epsilon}(x) .
$$

Using the previous proposition we immediately obtain

Proposition 5.3. Assume that 0 is a critical point of $p$ and $q$. If $p<\min \{\sqrt{n}$, $\left.\frac{n+2}{3}\right\}$, then

$$
\begin{aligned}
\int_{\mathbf{R}^{n}} f(x) v_{\epsilon}^{q(x)} d x & =f(0) K(n, p)^{-n}+f(0) A \epsilon^{2} \ln \epsilon+o\left(\epsilon^{2} \ln \epsilon\right), \\
\int_{\mathbf{R}^{n}} f(x)\left|\nabla v_{\epsilon}\right|^{p(x)} d x & =f(0) K(n, p)^{-n}+f(0) B \epsilon^{2} \ln \epsilon+o\left(\epsilon^{2} \ln \epsilon\right), \\
\int_{\mathbf{R}^{n}} f(x)\left|v_{\epsilon}\right|^{p(x)} d x & =f(0) C \epsilon^{p}+o\left(\epsilon^{p}\right),
\end{aligned}
$$

with

$$
\begin{aligned}
& A=-\frac{\Delta q(0)}{2 p^{*}} K(n, p)^{-n}\|U\|_{p^{*}}^{-p^{*}} \int_{\mathbf{R}^{n}}|x|^{2} U^{p^{*}} d x, \\
& B=-\frac{\Delta p(0)}{2 p} K(n, p)^{p-n}\|U\|_{p^{*}}^{-p} \int_{\mathbf{R}^{n}}|x|^{2}|\nabla U|^{p} d x, \\
& C=K(n, p)^{p-n}\|U\|_{p^{*}}^{-p}\|U\|_{p}^{p} .
\end{aligned}
$$


Using $v_{\epsilon}$ as a test-function in (1.11) we can see that there exists $t_{0}>1$ such that $J\left(t v_{\epsilon}\right)<0$ for $t>t_{0}$. Now if $p<2$, we can write

$$
f_{\epsilon}(t):=J\left(t v_{\epsilon}\right)=f_{0}(t)+\epsilon^{p} f_{1}(t)+o\left(\epsilon^{p}\right)
$$

$C^{1}$-uniformly in $t \in\left[0, t_{0}\right]$, with

$$
f_{0}(t)=K(n, p)^{-n}\left(\frac{t^{p}}{p}-\frac{t^{p^{*}}}{p^{*}}\right) \quad \text { and } \quad f_{1}(t)=\frac{1}{p} t^{p} h(0) C .
$$

Notice that $f_{0}$ reaches its maximum in $\left[0, t_{0}\right]$ at $t=1$. Moreover, it is a nondegenerate maximum since $f_{0}^{\prime \prime}(1)=\left(p-p^{*}\right) K^{-n} \neq 0$. It follows that $f_{\epsilon}$ reaches a maximum at $t_{\epsilon}=1+a \epsilon^{p}+o\left(\epsilon^{p}\right)$ for $a=-\frac{f_{1}^{\prime}(1)}{f_{0}^{\prime \prime}(1)}$. Hence

$$
\sup _{t>0} J\left(t v_{\epsilon}\right)=J\left(t_{\epsilon} v_{\epsilon}\right)=\frac{1}{n} K(n, p)^{-n}+f_{1}(1) \epsilon^{p}+o\left(\epsilon^{p}\right)
$$

Then if $h(0)<0$ we get $\sup _{t>0} J\left(t v_{\epsilon}\right)<\frac{1}{n} K(n, p)^{-n}$.

We now assume that $p \geq 2$. Then

$$
f_{\epsilon}(t)=J\left(t v_{\epsilon}\right)=f_{0}(t)+\tilde{f}_{1}(t) \epsilon^{2} \ln \epsilon+o\left(\epsilon^{2} \ln \epsilon\right),
$$

$C^{1}$-uniformly in $t \in\left[0, t_{0}\right]$, with

$$
\tilde{f}_{1}(t)=\frac{t^{p^{*}}}{p^{*}} A-\frac{t^{p}}{p} B
$$

As before $f_{\epsilon}$ reaches its maximum at $t_{\epsilon}=1+a \epsilon^{2} \ln \epsilon+o\left(\epsilon^{2} \ln \epsilon\right)$ with $a=-\frac{\tilde{f}_{1}^{\prime}(1)}{f_{0}^{\prime \prime}(1)}$. Hence

$$
\begin{aligned}
\sup _{t>0} J\left(t v_{\epsilon}\right) & =J\left(t_{\epsilon} v_{\epsilon}\right)=f_{0}(1)+\tilde{f}_{1}(1) \epsilon^{2} \ln \epsilon+o\left(\epsilon^{2} \ln \epsilon\right) \\
& =\frac{1}{n} K(n, p)^{-n}+\tilde{f}_{1}(1) \epsilon^{2} \ln \epsilon+o\left(\epsilon^{2} \ln \epsilon\right) .
\end{aligned}
$$

We thus need $\tilde{f}_{1}(1)<0$, i.e.,

$$
-\Delta p(0)<-\Delta q(0)\left(p / p^{*}\right)^{2} D(n, p),
$$

where

$$
D(n, p):=\frac{\int_{\mathbf{R}^{n}}|\nabla U|^{p} d x \int_{\mathbf{R}^{n}}|x|^{2} U^{p^{*}} d x}{\int_{\mathbf{R}^{n}} U^{p^{*}} d x \int_{\mathbf{R}^{n}}|x|^{2}|\nabla U|^{p} d x} .
$$

Since 0 is a local maximum of $q$ and a local minimum of $p$ we already know that (1.12) holds. Then if one of the two inequalities in (1.12) is strict we see that (5.5) holds.

This ends the proof of Theorem 1.3.

As a final remark, we notice that we can compute $D(n, p)$ exactly. To do this let

$$
I_{p}^{q}:=\int_{0}^{\infty} t^{q-1}(1+t)^{-p} d t=B(q, p-q)=\frac{\Gamma(q) \Gamma(p-q)}{\Gamma(p)},
$$


where $B(x, y):=\int_{0}^{\infty} t^{x-1}(1+t)^{-x-y} d t$ is the Beta function. This formula can be found, for instance, in [3]. Passing to spherical coordinates and then performing the change of variable $t=r^{\frac{p}{p-1}}, d r=\frac{p-1}{p} t^{-\frac{1}{p}} d t$, we obtain

$$
\begin{aligned}
& \int_{\mathbf{R}^{n}} U^{p^{*}} d x=U_{n-1} \frac{p-1}{p} I_{n}^{n \frac{p-1}{p}}, \\
& \int_{\mathbf{R}^{n}}|x|^{2} U^{p^{*}} d x=\omega_{n-1} \frac{p-1}{p} I_{n}^{n \frac{p-1}{p}-\frac{2}{p}+2}, \\
& \int_{\mathbf{R}^{n}}|\nabla U|^{p} d x=\omega_{n-1} \frac{p-1}{p}\left(\frac{n-p}{p-1}\right)^{p} I_{n}^{n \frac{p-1}{p}+1}, \\
& \int_{\mathbf{R}^{n}}|x|^{2}|\nabla U|^{p} d x=\omega_{n-1} \frac{p-1}{p}\left(\frac{n-p}{p-1}\right)^{p} I_{n}^{n \frac{p-1}{p}-\frac{2}{p}+3} .
\end{aligned}
$$

Then

$$
D(n, p)=\frac{I_{n}^{\frac{n(p-1)}{p}+1} I_{n}^{\frac{n(p-1)}{p}-\frac{2}{p}+2}}{I_{n}^{\frac{n(p-1)}{p}} I_{n}^{\frac{n(p-1)}{p}-\frac{2}{p}+3}}=\frac{n}{n-p} \frac{(n-p)-2(p-1)}{n+2},
$$

where we used that

$$
I_{p}^{q+1}=\frac{q}{p-q-1} I_{p}^{q}
$$

which follows from (5.6) and the formula $\Gamma(z+1)=z \Gamma(z)$.

\section{Proof of Proposition 5.1}

As 0 is a local minimum of $p(\cdot)$ we can assume that $p_{2 \delta}^{-}:=\min _{x \in B_{2 \delta}} p(x)=p$.

6.1. Proof of (5.1). We first write

$$
\int_{\mathbf{R}^{n}} f(x) u_{\epsilon}(x)^{q(x)} d x=\int_{B_{2 \delta} \backslash B_{\epsilon^{1 / p}}} f(x) u_{\epsilon}^{q(x)} d x+\int_{B_{\epsilon^{1 / p}}} f(x) u_{\epsilon}(x)^{q(x)} d x=I_{1}(\epsilon)+I_{2}(\epsilon) .
$$

Since $u_{\epsilon}(x) \leq 1$ if $|x| \geq \epsilon^{1 / p}$, we have, letting $q_{2 \delta}^{-}:=\min _{B_{2 \delta}} q$ that

$$
\begin{aligned}
I_{1}(\epsilon) & \leq\|f\|_{L^{\infty}\left(B_{2 \delta}\right)} \int_{B_{2 \delta} \backslash B_{\epsilon^{1 / p}}} u_{\epsilon}(x)^{q_{2 \delta}^{-}} d x \\
& \leq\|f\|_{L^{\infty}\left(B_{2 \delta}\right)} \epsilon^{n-\frac{n-p}{p} q_{2 \delta}^{-}} \int_{\mathbf{R}^{n} \backslash B_{\epsilon^{-(p-1) / p}}} U(x)^{q_{2 \delta}^{-}} d x,
\end{aligned}
$$

where the integral in the right hand side can be bounded by

$$
C \int_{\epsilon^{-(p-1) / p}}^{+\infty}\left(1+r^{\frac{p}{p-1}}\right)^{-\frac{n-p}{p} q_{2 \delta}^{-}} r^{n-1} d r \leq C \int_{\epsilon^{-(p-1) / p}}^{+\infty} r^{-1+n-\frac{n-p}{p-1} q_{2 \delta}^{-}} d r \leq C \epsilon^{-n \frac{p-1}{p}+\frac{n-p}{p} q_{2 \delta}^{-}} .
$$

Hence $I_{1}(\epsilon) \leq C \epsilon^{n / p}$ so that

$$
\begin{aligned}
\int_{\mathbf{R}^{n}} f(x) u_{\epsilon}(x)^{q(x)} d x & =\int_{B_{\epsilon^{1 / p}}} f(x) u_{\epsilon}(x)^{q(x)} d x+O\left(\epsilon^{n / p}\right) \\
& =\int_{B_{\epsilon^{-(p-1) / p}}} f(\epsilon x) \epsilon^{n-q(\epsilon x) \frac{n-p}{p}} U(x)^{q(\epsilon x)} d x+O\left(\epsilon^{n / p}\right) .
\end{aligned}
$$


As $\nabla q(0)=0$, we get

$$
q(\epsilon x)=q(0)+\frac{1}{2} \epsilon^{2}\left(D^{2} q(0) x, x\right)+o\left(\epsilon^{2}|x|^{2}\right),
$$

with $q(0)=p(0)^{*}=p^{*}$, so

$$
\begin{aligned}
\int_{\mathbf{R}^{n}} f(x) u_{\epsilon}(x)^{q(x)} d x= & A_{0}(\epsilon)+A_{1}(\epsilon) \epsilon^{2} \ln \epsilon+\int_{B_{\epsilon^{-(p-1) / p}}} O\left(\epsilon^{2} \ln \epsilon\right)|x|^{2} U(x)^{p^{*}} d x \\
& +\epsilon \int_{B_{\epsilon^{-(p-1) / p}}} U(x)^{p^{*}} \nabla f(0) \cdot x d x+O\left(\epsilon^{n / p}\right) \\
= & A_{0}(\epsilon)+A_{1}(\epsilon) \epsilon^{2} \ln \epsilon+o\left(\epsilon^{2} \ln \epsilon\right)+O\left(\epsilon^{n / p}\right),
\end{aligned}
$$

where $A_{0}(\epsilon)$ and $A_{1}(\epsilon)$ are the same as $A_{0}$ and $A_{1}$ except that we integrate over $B_{\epsilon^{-(p-1) / p}}$ instead of $\mathbf{R}^{n}$ and we have used the fact that

$$
\int_{B_{\epsilon^{-}(p-1) / p}} U(x)^{p^{*}} \nabla f(0) \cdot x d x=0
$$

since $U$ is radially symmetric. We have

$$
\begin{aligned}
\left|A_{0}(\epsilon)-A_{0}\right| & \leq C \int_{\mathbf{R}^{n} \backslash B_{\epsilon^{-}(p-1) / p}} U(x)^{p^{*}} d x \leq C \int_{\epsilon^{-(p-1) / p}}^{+\infty}\left(1+r^{\frac{p}{p-1}}\right)^{-n} r^{n-1} d r \\
& \leq C \int_{\epsilon^{-(p-1) / p}}^{+\infty} r^{\frac{-n p}{p-1}+n-1} d r \leq C \epsilon^{\frac{n}{p}} .
\end{aligned}
$$

If $p<(n+2) / 2$, we can estimate

$$
\begin{aligned}
\left|A_{1}(\epsilon)-A_{1}\right| & \leq C \int_{\mathbf{R}^{n} \backslash B_{\epsilon^{-(p-1) / p}}}|x|^{2} U(x)^{p^{*}} d x \\
& \leq C \int_{\epsilon^{-(p-1) / p}}^{+\infty}\left(1+r^{\frac{p}{p-1}}\right)^{-n} r^{n+1} d r \leq C \epsilon^{\frac{n+2-2 p}{p}} .
\end{aligned}
$$

We thus have

$$
\int_{\mathbf{R}^{n}} f(x) u_{\epsilon}(x)^{q(x)} d x-A_{0}-A_{1} \epsilon^{2} \ln \epsilon=O\left(\epsilon^{n / p}\right)+o\left(\epsilon^{2} \ln \epsilon\right),
$$

which reduces to (5.1) if we assume that $p \leq n / 2$.

6.2. Proof of (5.3). As before,

$$
\int_{\mathbf{R}^{n}} f(x) u_{\epsilon}^{p(x)} d x=\int_{B_{\epsilon^{1 / p}}} f(x) u_{\epsilon}^{p(x)} d x+\int_{B_{2 \delta} \backslash B_{\epsilon^{1 / p}}} f(x) u_{\epsilon}^{p(x)} d x
$$

where, noticing that $p=p_{2 \delta}^{-}$, the 2 nd integral in the right hand side can be bounded by

$$
\int_{B_{2 \delta} \backslash B_{\epsilon^{1 / p}}} u_{\epsilon}^{p} d x \leq C \epsilon^{p} \int_{\epsilon^{1 / p-1}}^{\infty}\left(1+r^{\frac{p}{p-1}}\right)^{p-n} r^{n-1} d r \leq C \epsilon^{p} \epsilon^{\frac{n-p^{2}}{p}}=C \epsilon^{\frac{n}{p}}
$$

if $p^{2}<n$. Then

$$
\int_{\mathbf{R}^{n}} f(x) u_{\epsilon}^{p(x)} d x=\int_{B_{\epsilon^{1 / p}}} f(x) u_{\epsilon}^{p(x)} d x+O\left(\epsilon^{\frac{n}{p}}\right)
$$


$=\int_{B_{\epsilon^{1 / p-1}}} f(\epsilon x) \epsilon^{n-\frac{n-p}{p} p(\epsilon x)} U(x)^{p(\epsilon x)} d x+O\left(\epsilon^{\frac{n}{p}}\right)=\epsilon^{p} f(0) \int_{\mathbf{R}^{n}} U(x)^{p} d x+o\left(\epsilon^{p}\right)$.

6.3. Proof of (5.2). We first write

$\int_{\mathbf{R}^{n}} f(x)\left|\nabla u_{\epsilon}\right|^{p(x)} d x=\int_{\mathbf{R}^{n}} f(x)\left|\eta \nabla U_{\epsilon}+U_{\epsilon} \nabla \eta\right|^{p(x)} d x=\int_{\mathbf{R}^{n}} f(x)\left|\eta \nabla U_{\epsilon}\right|^{p(x)} d x+R_{\epsilon}$, where, using the inequality

$$
|| a+\left.b\right|^{q}-|a|^{q} \mid \leq C\left(|b|^{q}+|b||a|^{q-1}\right),
$$

(the constant $C$ being uniform in $q$ for $q$ in a bounded interval of $[0,+\infty)$ ) we can estimate

$\left|R_{\epsilon}\right| \leq C\left[\int_{B_{2 \delta} \backslash B_{\delta}}|\nabla \eta|^{p(x)} U_{\epsilon}^{p(x)} d x+\int_{B_{2 \delta} \backslash B_{\delta}}|\nabla \eta| U_{\epsilon}(x)\left|\nabla U_{\epsilon}\right|^{p(x)-1} d x\right]=C\left[I_{1}(\epsilon)+I_{2}(\epsilon)\right]$.

Since $U_{\epsilon} \leq 1$ in $\mathbf{R}^{n} \backslash B_{\delta}$ for $\epsilon$ small, we can bound $I_{1}(\epsilon)$ as before by

$$
I_{1}(\epsilon) \leq C \int_{B_{2 \delta} \backslash B_{\delta}} U_{\epsilon}^{p} d x \leq C \epsilon^{p} \int_{\mathbf{R}^{n} \backslash B_{\delta / \epsilon}} U^{p} d x \leq C \epsilon^{p} \epsilon^{\frac{n-p^{2}}{p-1}}=C \epsilon^{\frac{n-p}{p-1}},
$$

if $p^{2}<n$. Since $\left|\nabla U_{\epsilon}\right| \leq 1$ in $\mathbf{R}^{n} \backslash B_{\delta}$ for $\epsilon$ small, we also have

$$
\begin{aligned}
I_{2}(\epsilon) & \leq C \int_{\mathbf{R}^{n} \backslash B_{\delta}} U_{\epsilon}(x)\left|\nabla U_{\epsilon}\right|^{p-1} d x \leq C\left\|U_{\epsilon}\right\|_{L^{p}\left(\mathbf{R}^{n} \backslash B_{\delta}\right)}\left\|\nabla U_{\epsilon}\right\|_{L^{p}\left(\mathbf{R}^{n} \backslash B_{\delta}\right)}^{p-1} \\
& \leq C \epsilon^{\frac{n-p}{p(p-1)}}\left\|\nabla U_{\epsilon}\right\|_{L^{p}\left(\mathbf{R}^{n} \backslash B_{\delta}\right)}^{p-1},
\end{aligned}
$$

with, since $\left|U^{\prime}(r)\right| \sim r^{-\frac{n-1}{p-1}}$ as $r \sim+\infty$,

$$
\int_{\mathbf{R}^{n} \backslash B_{\delta}}\left|\nabla U_{\epsilon}\right|^{p} d x \leq C \int_{\delta / \epsilon}^{+\infty}\left|U^{\prime}(r)\right|^{p} r^{n-1} d r \leq C \epsilon^{\frac{n-p}{p-1}}
$$

It follows that $I_{2}(\epsilon)=O\left(\epsilon^{\frac{n-p}{p-1}}\right)$ and then $R_{\epsilon}=O\left(\epsilon^{\frac{n-p}{p-1}}\right)$. Independently, since

$$
\left|\nabla U_{\epsilon}(x)\right|=\frac{n-p}{p-1} \epsilon^{-n / p}\left(\frac{|x|}{\epsilon}\right)^{\frac{1}{p-1}}\left(1+\left(\frac{|x|}{\epsilon}\right)^{\frac{p}{p-1}}\right)^{-n / p}
$$

we have

$$
\left|\nabla U_{\epsilon}(x)\right|<1 \text { for }|x|>C_{p} \epsilon^{\frac{n-p}{p(n-1)}}, \quad C_{p}=\left(\frac{n-p}{p-1}\right)^{\frac{p-1}{n-1}} .
$$

Taking some constant $C>C_{p}$, we thus write

$$
\begin{aligned}
\int_{\mathbf{R}^{n}} f(x)\left|\nabla u_{\epsilon}\right|^{p(x)} d x= & \int_{B}{ }_{C \epsilon}^{\frac{n-p}{p(n-1)}} f(x)\left|\nabla U_{\epsilon}\right|^{p(x)} d x \\
& +\int_{\mathbf{R}^{n} \backslash B} \underset{C \epsilon}{\frac{n-p}{p(n-1)}} f(x)\left|\nabla U_{\epsilon}\right|^{p(x)} d x+O\left(\epsilon^{\frac{n-p}{p-1}}\right) .
\end{aligned}
$$


Since $\left|\nabla U_{\epsilon}(x)\right|<1$ in $\mathbf{R}^{n} \backslash B_{C \epsilon^{\frac{n-p}{p(n-1)}}}$, we can bound the second integral on the right hand side by

$C \int_{\mathbf{R}^{n} \backslash B}\left|\nabla U_{\epsilon \epsilon}\right|^{p} d x \leq\left. C \int_{\epsilon}^{\frac{n-p}{p(n-1)}}\right|^{+\infty} \frac{n(p-1)}{p(n-1)} r^{\frac{p}{p-1}}\left(1+r^{\frac{p}{p-1}}\right)^{-n} r^{n-1} d r \leq C \epsilon^{\frac{n(n-p)}{p(n-1)}}=o\left(\epsilon^{\frac{n-p}{p-1}}\right)$.

Hence

$$
\begin{aligned}
\int_{\mathbf{R}^{n}} f(x)\left|\nabla u_{\epsilon}\right|^{p(x)} d x & =\int_{B} \underset{C \epsilon}{\frac{n-p}{p(n-1)}} f(x)\left|\nabla U_{\epsilon}\right|^{p(x)} d x+O\left(\epsilon^{\frac{n-p}{p-1}}\right) \\
& =B_{0}(\epsilon)+B_{1}(\epsilon) \epsilon^{2} \ln \epsilon+o\left(\epsilon^{2} \ln \epsilon\right)+O\left(\epsilon^{\frac{n-p}{p-1}}\right),
\end{aligned}
$$

where $B_{0}(\epsilon)$ and $B_{1}(\epsilon)$ are the same as $B_{0}, B_{1}$ but integrating over $B_{\epsilon-\frac{n(p-1)}{p(n-1)}}$ instead of $\mathbf{R}^{n}$. Again, as in the computation of (5.1), the term involving $\nabla f(0)$ vanishes for symmetry reasons.

Since $\left|U^{\prime}(r)\right|^{p} \sim r^{\frac{p(1-n)}{p-1}}$ as $r \sim+\infty$, we have

$$
\begin{aligned}
& \left|B_{0}-B_{0}(\epsilon)\right| \leq C \int_{\mathbf{R}^{n} \backslash B}|\nabla U|^{p} d x \leq C \int_{\epsilon^{-\frac{n(p-1)}{p(n-1)}}}^{+\infty} r^{\frac{p-n}{p-1}-1} d r \leq C \epsilon^{\frac{n(n-p)}{p(n-1)}}=o\left(\epsilon^{\frac{n-p}{p-1}}\right), \\
& \left|B_{1}-B_{1}(\epsilon)\right| \leq C \int_{\mathbf{R}^{n} \backslash B}|x|^{2}|\nabla U|^{p} d x \leq C \epsilon^{\frac{n(n-3 p+2)}{p(n-1)}} \quad \text { if } p<\frac{n+2}{3} .
\end{aligned}
$$

Hence, if $p<\frac{n+2}{3}$, we have

$$
\int_{\mathbf{R}^{n}} f(x)\left|\nabla u_{\epsilon}\right|^{p(x)} d x-B_{0}-B_{1} \epsilon^{2} \ln \epsilon=o\left(\epsilon^{2} \ln \epsilon\right) .
$$

Acknowledgements. This work was partially supported by Universidad de Buenos Aires under grant X078 and by CONICET (Argentina) PIP 5478/1438. A. Silva is a fellow of CONICET.

\section{References}

[1] Alves, C. O., and M. A. S. Souto: Existence of solutions for a class of problems in $\mathbf{R}^{N}$ involving the $p(x)$-Laplacian. - In: Contributions to nonlinear analysis, Progr. Nonlinear Differential Equations Appl. 66, Birkhäuser, Basel, 2006, 17-32.

[2] Aubin, T.: Problèmes isopérimétriques et espaces de Sobolev. - J. Differential Geom. 11:4, 1976, 573-598.

[3] Beals, R., and R. Wong: Special functions. - Cambridge Stud. Adv. Math. 126, Cambridge Univ. Press, Cambridge, 2010.

[4] BRÉZIS, H., and E. LIEB: A relation between pointwise convergence of functions and convergence of functionals. - Proc. Amer. Math. Soc. 88:3, 1983, 486-490.

[5] Brézis, H., and L. Nirenberg: Positive solutions of nonlinear elliptic equations involving critical Sobolev exponents. - Comm. Pure Appl. Math. 36:4, 1983, 437-477.

[6] Cabada, A., and R. L. Pouso: Existence theory for functional $p$-Laplacian equations with variable exponents. - Nonlinear Anal. 52:2, 2003, 557-572.

[7] Chen, Y., S. Levine, and M. RAO: Variable exponent, linear growth functionals in image restoration. - SIAM J. Appl. Math. 66:4, 2006, 1383-1406 (electronic). 
[8] Diening, L., P. Harjulehto, P. Hästö, and M. RŮŽIČKa: Lebesgue and Sobolev spaces with variable exponents. - Lecture Notes in Math. 2017, Springer, Heidelberg, 2011.

[9] Druet, O.: Generalized scalar curvature type equations on compact Riemannian manifolds. Proc. Roy. Soc. Edinburgh Sect. A 130:4, 2000, 767-788.

[10] FAn, X.-L., and Q.-H. Zhang: Existence of solutions for $p(x)$-Laplacian Dirichlet problem. Nonlinear Anal. 52:8, 2003, 1843-1852.

[11] FAn, X., and D. ZhaO: On the spaces $L^{p(x)}(\Omega)$ and $W^{m, p(x)}(\Omega)$. - J. Math. Anal. Appl. 263:2, 2001, 424-446.

[12] Fernández Bonder, J., N. Saintier, and A. Silva: On the Sobolev embedding Theorem for variable exponent spaces in the critical range. - J. Differential Equations 253:5, 2012, 16041620.

[13] Fernández Bonder, J., N. SAintier, and A. Silva: On the Sobole trace Theorem for variable exponent spaces in the critical range. - In preparation.

[14] Fernández Bonder, J., and A. Silva: Concentration-compactness principle for variable exponent spaces and applications. - Electron. J. Differential Equations 141:18, 2010.

[15] FU, Y.: The principle of concentration compactness in $L^{p(x)}$ spaces and its application. Nonlinear Anal. 71:5-6, 2009, 1876-1892.

[16] FU, Y., and X. ZHANG: Multiple solutions for a class of $p(x)$-Laplacian equations in involving the critical exponent. - Proc. R. Soc. Lond. Ser. A Math. Phys. Eng. Sci. 466:2118, 2010, $1667-1686$.

[17] Guedda, M., and L. Véron: Quasilinear elliptic equations involving critical Sobolev exponents. - Nonlinear Anal. 13:8, 1989, 879-902.

[18] KováC̆IK, O., and J. RÁkosník: On spaces $L^{p(x)}$ and $W^{k, p(x)}$. - Czechoslovak Math. J. 41(116):4, 1991, 592-618.

[19] Lions, P.-L., F. Pacella, and M. Tricarico: Best constants in Sobolev inequalities for functions vanishing on some part of the boundary and related questions. - Indiana Univ. Math. J. 37:2, 1988, 301-324.

[20] MinĂIlescu, M.: Elliptic problems in variable exponent spaces. - Bull. Austral. Math. Soc. 74:2, 2006, 197-206.

[21] MihăIlescu, M.: On a class of nonlinear problems involving a $p(x)$-Laplace type operator. Czechoslovak Math. J. 58(133):1, 2008, 155-172.

[22] MihĂILESCU, M., and V. RĂDULESCU: On a nonhomogeneous quasilinear eigenvalue problem in Sobolev spaces with variable exponent. - Proc. Amer. Math. Soc. 135:9, 2007, 2929-2937 (electronic).

[23] Mizuta, Y., T. Ohno, T. Shimomura, and N. Shioji: Compact embeddings for Sobolev spaces of variable exponents and existence of solutions for nonlinear elliptic problems involving the $p(x)$-Laplacian and its critical exponent. - Ann. Acad. Sci. Fenn. Math. 35:1, 2010, 115-130.

[24] PohožAev, S. I.: On the eigenfunctions of the equation $\Delta u+\lambda f(u)=0$. - Dokl. Akad. Nauk SSSR 165, 1965, 36-39.

[25] RŮŽIČKA, M.: Electrorheological fluids: modeling and mathematical theory. - Lecture Notes in Math. 1748, Springer-Verlag, Berlin, 2000.

[26] SAintier, N.: Asymptotic estimates and blow-up theory for critical equations involving the p-Laplacian. - Calc. Var. Partial Differential Equations 25:3, 2006, 299-331.

[27] Silva, A.: Multiple solutions for the $p(x)$-Laplace operator with critical growth. - Adv. Nonlinear Stud. 11:1 2011, 63-75.

[28] Talenti, G.: Best constant in Sobolev inequality. - Ann. Mat. Pura Appl. (4) 110, 1976, $353-372$.

Received 11 April 2012 